\section{InP-based single-photon detector arrays with asynchronous readout integrated circuits}

\author{
James B. Glettler, Pablo I. Hopman, Simon Verghese, \\ Joseph C. Aversa, Larry M. Candell, \\ Joseph P. Donnelly, Erik K. Duerr, \\ Jonathan P. Frechette, Joseph E. Funk, Z. L. Liau, \\ K. A. Mclntosh, Leonard J. Mahoney, Karen M. Molvar, \\ Douglas C. Oakley, E. J. Ouellette, Gary M. Smith, \\ and Christopher J. Vineis \\ Massachusetts Institute of Technology, Lincoln Laboratory, \\ Lexington, Massachusetts 02420
}

\begin{abstract}
We have developed and demonstrated a highduty-cycle asynchronous InGaAsP-based photon counting detector system with near-ideal Poisson response, roomtemperature operation, and nanosecond timing resolution for near-infrared applications. The detector is based on an array of Geiger-mode avalanche photodiodes coupled to a custom integrated circuit that provides for lossless readout via an asynchronous, nongated architecture. We present results showing Poisson response for incident photon flux rates up to 10 million photons per second and multiple photons per 3-ns timing bin. () 2008 Society of Photo-Optical Instrumentation Engineers.

[DOI: 10.1117/1.2992138]
\end{abstract}

Subject terms: focal plane array; readout integrated circuit; avalanche photodiode; InGaAsP; photon counting.

Paper 080440LR received Jul. 7, 2008; revised manuscript received Aug. 21, 2008; accepted for publication Aug. 21, 2008; published online Oct. 9, 2008.

Applications to date using Geiger-mode avalanche photodiodes (GM APDs), such as three-dimensional laser $\operatorname{radar}^{1}$ and quantum-key distribution, ${ }^{2}$ use gated Geigermode operation in which the diode is biased above its breakdown voltage for a fixed duration at a regular interval. ${ }^{3}$ This gated mode of operation, however, limits the utility of GM APD detectors to low-duty-cycle applications, where the gate is synchronous with the optical signal. We present an approach to expand the utility of GM APDs to applications requiring high duty cycle and asynchronous operation. ${ }^{4}$ We demonstrate that by operating an array of GM-APD detectors asynchronously, the aggregated array response approaches that of an ideal photon-counting detector, maintaining the Poisson statistics of the input beam.

In asynchronous mode an individual APD is overbiased until an avalanche event (firing) occurs, which causes the bias to be reduced below breakdown for a fixed holdoff time before rearming. During the avalanche process carriers populate in-band traps. If the device is overbiased while these traps are filled, it can lead to spontaneous firing when the traps depopulate. To avoid this undesirable premature firing, known as afterpulsing, the bias must be held below breakdown for a holdoff time to allow for the traps to clear prior to rearming. ${ }^{5}$ In this asynchronous mode of operation, the device spends the majority of the time in the overbiased

0091-3286/2008/\$25.00 @ 2008 SPIE state and is only brought below breakdown to quench the avalanche and clear the traps, maximizing the time available to detect photons. This is in contrast to gated mode, where the device typically is only biased for a few percent of the time.

The typical minimum holdoff time for an InP-based near-infrared APD at room temperature is on the order of $1 \mu \mathrm{s},{ }^{6}$ which would limit an operating asynchronous APD to an average incident photon flux around $10^{6}$ photons/s. As the rate increases beyond $10^{6}$ photons/s the detector spends the majority of the time in holdoff, where incident photons are blocked from being detected. For a simple Poisson source, the probability that a single APD element is blocked can be calculated as the fraction of time the APD is in holdoff:

$P_{\text {blocked,single }}=\frac{t_{\mathrm{ho}}}{t_{\mathrm{ho}}+\left\langle t_{\gamma}\right\rangle}$,

where $\left\langle t_{\gamma}\right\rangle$ is the mean time to fire after rearm, which is simply the reciprocal of the expectation value of the perpixel firing rate, and $t_{\text {ho }}$ is the holdoff time. By distributing the incident photon beam over an array of APDs and digitally aggregating the output of each independent element in the array, the self-blocking effect can be mitigated. Equation (1) can then be extended to the probability a photon is blocked, using the aggregated output of an array of $N$ independent elements:

$P_{\text {blocked,array }}=\frac{1}{1+\left[t_{\mathrm{ho}}\left(\lambda_{\text {Source }} / N+\lambda_{\mathrm{DCR}}\right)\right]^{-1}}$,

where $\lambda_{\text {DCR }}$ is the dark count rate per pixel, $\lambda_{\text {Source }}$ is the mean photon arrival rate across the entire array, and $N$ is the number of pixels in the array. The expression in Eq. (2) assumes uniform illumination but can easily be extended to cover the nonuniform case. By increasing the array size, the total incident flux allowed for a given blocking probability increases; however, the total dark-count contribution to the aggregate output scales with the number of pixels used in the array.

The performance metrics specific to an arrayed detector operated in this mode are the aggregated dark count of the array $\left(\mathrm{DCR}=N \lambda_{\mathrm{DCR}}\right)$ and the probability an incident photon is blocked [Eq. (2)]. The product of $\lambda_{\mathrm{DCR}}$ and $t_{\mathrm{ho}}$ uniquely fixes the total DCR and blocking performance parameters and is an effective figure of merit. These parameters are strongly temperature-dependent, decreasing the temperature results in reduced dark count rate but increases trap lifetime and requires the holdoff time to increase. One can allow a longer holdoff time by reducing the singlepixel dark count rate or increasing the number of pixels.

For asynchronous array operation, each pixel detects and resets asynchronously, thereby randomly sampling the optical source. For large arrays, the response of the aggregated output approaches an ideal photon-counting detector with a Poisson response. The Poisson-limited output is a fundamental requirement for many applications. ${ }^{7}$ In particular, the detector concept described in this paper has been an enabling technology for highly efficient photoncounting communication links. ${ }^{8,9}$

There were three challenges in implementing this array detector concept. The first was to develop APD detectors with sufficiently low $t_{\mathrm{ho}} \lambda_{\mathrm{DCR}}$ and good detection efficiency. 

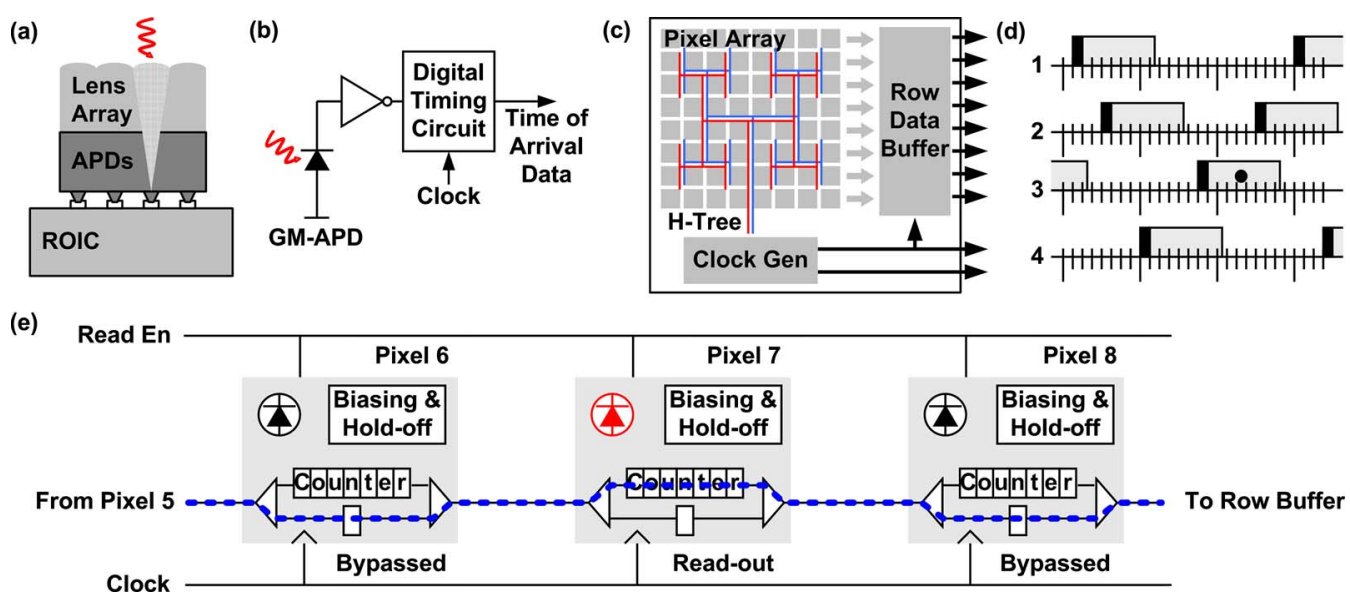

Fig. 1 (a) Physical cross section of photon-counting stacked hybrid. (b) Connection of APD to timing circuit. (c) Block diagram of readout integrated circuit (ROIC). (d) Representative timing diagram, not to scale. (e) Section of ROIC pixel row circuit. Pixel 7 has fired and is read out without interfering with unfired pixels.

We used back-illuminated InGaAsP/InP detectors sensitive to 1.06- $\mu \mathrm{m}$ light, following Lincoln Laboratory APD development. ${ }^{3,6,10}$ Uncooled, these detectors operate at about $50 \%$ photon detection efficiency and have a $40-\mathrm{kHz}$ dark count rate with a $3-\mu$ s holdoff. Each APD has a $15-\mu \mathrm{m}$ active diameter, and the detectors are arrayed in an $8 \times 8$ grid on a $100-\mu$ m pitch. ${ }^{10}$ The second challenge was then to efficiently couple light onto the active area of each APD. An antireflection-coated gallium phosphide microlens array was hybridized to the APD array to increase the fill factor to about $80 \%$, resulting in an effective $40 \%$ photon detection efficiency. The third challenge was to operate each APD asynchronously. A custom readout integrated circuit (ROIC) containing all necessary APD biasing and arrival-time detection functions is indium-bump bonded to the APD array. This complete stack is depicted in Fig. 1(a).

The ROIC was fabricated using a commercial $0.35-\mu \mathrm{m}$ complementary metal-oxide semiconductor (CMOS) process. Each APD in the array operates as an independent pixel. Geiger-mode operation enables each APD to directly interface with CMOS logic, as in Fig. 1(b). Each pixel has circuitry for APD biasing, variable avalanche detection with active quenching, a free-running counter to time avalanche events, and a holdoff time counter. An avalanche causes the free-running counter to stop, thereby recording the time of photon arrival, and starts the holdoff time counter. When the holdoff elapses, the APD is biased asynchronously. The pixels in the array are organized into rows as depicted in Fig. 1(c). A clock distribution tree is used on chip to guarantee uniform timing. Periodically, the time-ofavalanche counter values for pixels that have been triggered are shifted out of the array and off the ROIC. The data output process does not affect nontriggered pixels [see Fig. $1(\mathrm{e})]$ and completes during the holdoff time. When the output is sent to an external field-programmable gate array, the complete photon arrival-time history of each APD pixel is decoded.

Figure 1(d) shows a notional timing diagram for four illuminated pixels, one pixel per row, with time increasing along the $x$ axis. Each black rectangle corresponds to an APD avalanche, followed by a gray bar representing the holdoff time when the APD is unbiased. A photon that hits an unbiased pixel will still be blocked, as represented by the black circle in the figure. The diagram shows how a firing in one pixel does not block the detection of photons in any other pixel. By exploiting the holdoff time, this architecture has zero overhead for data readout, which is necessary for high overall detection efficiency and saturation power.

Figure 2(a) is a measured histogram of the time between APD dark counts (interarrival time) for a single unilluminated pixel extracted from the aggregate array data. The ROIC was operated at $311 \mathrm{MHz}$, resulting in a holdoff time of $3.2 \mu \mathrm{s}$, although the APDs support shorter holdoff times. The holdoff time manifests as an absence of photon arrivals before $3.2 \mu \mathrm{s}$. This single pixel has a $38-\mathrm{kHz}$ dark count rate at $30{ }^{\circ} \mathrm{C}$, consistent with single-device results reported

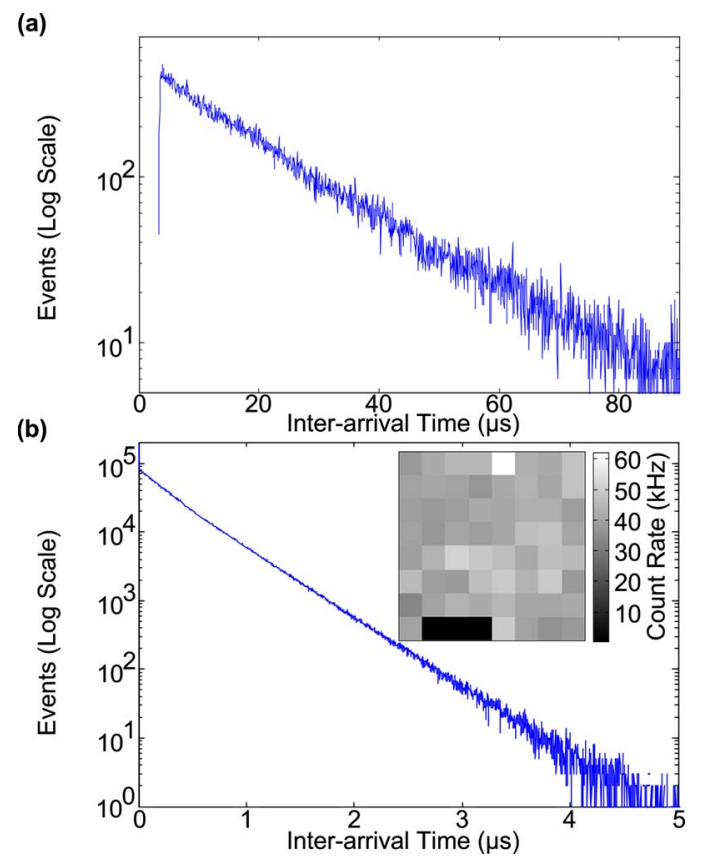

Fig. 2 Interarrival-time histograms of unilluminated APD firings for (a) a single detector and (b) 64 detectors. Inset: map of average dark count rate per detector. 


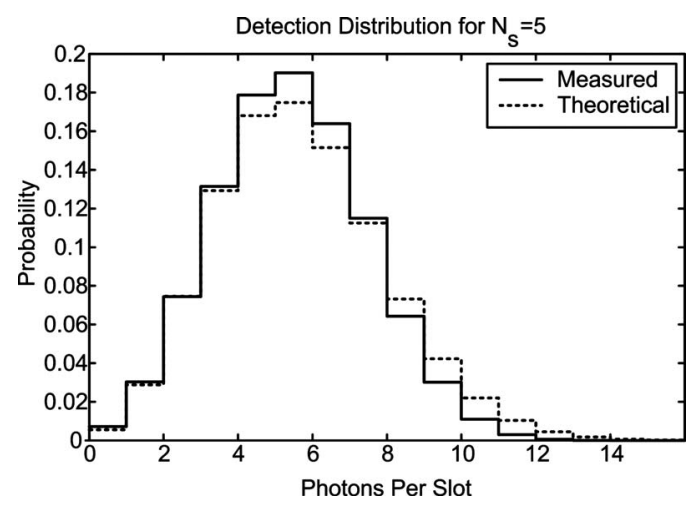

Fig. 3 Probability distribution of number of detected APD firing events per time slot with pulsed laser illumination at a fixed power level, showing multiphoton sensitivity.

by Jensen et al. ${ }^{6}$ This comparison shows that the array concept causes no additional artifacts in detector behavior. An ideal photon-counting detector with a Poisson-distributed input maintains that distribution on the output. The interarrival-time histogram would then be an exponential distribution, but because of the holdoff time the response is clearly not Poisson.

When the APD data from multiple pixels are digitally aggregated, as in Fig. 2(b), the asynchronous operation of the ROIC mitigates the holdoff time of any one pixel. In this case, measured interarrival times can be zero when two or more APDs fire within a single timing slot. The interarrival histogram follows an exponential distribution over many decades and demonstrates that a near-ideal photoncounting detector can be constructed using multiple asynchronous GM APDs. The inset in Fig. 2(b) is a map of dark count rates per pixel across the $8 \times 8$ array. Three pixels along the bottom row are disabled due to fabrication defects, and the remaining 61 pixels are relatively uniform with an average dark count rate of approximately $40 \mathrm{kHz}$.

A test setup was constructed to illuminate the photoncounting array with subnanosecond laser pulses from a $1.06-\mu \mathrm{m}$ source. We measured an APD detection efficiency of $40 \%$, including micro-lens losses-consistent with previous measurements of single devices. ${ }^{11}$ About $3 \%$ of detected photon events were recorded with a time of arrival off by one clock cycle due to timing jitter in the detector and ROIC.

Figure 3 shows the measured probability distribution for the detected number of APD firings per laser pulse, compared with theoretical Poisson distribution. The source laser power was adjusted to produce 5 photon-induced avalanches on average in a single 3.2-ns time slot. The histogram data matches a Poisson distribution closely. This demonstrates that the array is capable of measuring multiphoton events with wide dynamic range and response approaching an ideal photon-counting detector. The Poisson response of this asynchronous APD array, given high flux rates combined with the high photon efficiency and timing resolution, is unprecedented performance for a photon-counting near-infrared detector. Figure 4 shows a close-up picture of a fully packaged detector array in a test setup.

In summary, we operated an $8 \times 8$ photon-counting detector array at flux levels up to 10 million photons per sec-

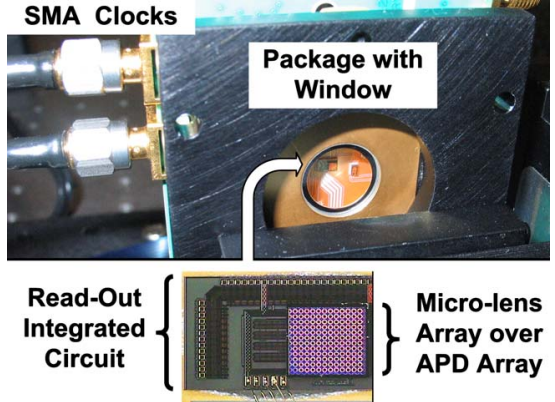

Fig. 4 Fully packaged photon-counting detector in test setup. Inset: Completed detector is about $3 \times 2 \mathrm{~mm}$.

ond. We showed how the aggregated output of the asynchronous mode CMOS circuit mitigates blocking of individual pixels and approaches the Poisson response of an ideal photon detector with high sensitivity and dynamic range. The $1.06-\mu \mathrm{m}$ detectors operated at room temperature with 3.2-ns timing resolution, $40 \%$ detection efficiency, and an average of $40 \mathrm{kHz}$ of dark counts per pixel.

\section{Acknowledgment}

This work was sponsored by the National Aeronautics and Space Administration under Air Force contract No. FA8721-05-C-0002. Opinions, interpretations, conclusions, and recommendations are those of the author and are not necessarily endorsed by the United States Air Force.

\section{References}

1. B. F. Aull, A. H. Loomis, D. J. Young, R. M. Heinrichs, B. J. Felton, P. J. Daniels, and D. J. Landers, "Geiger-mode avalanche photodiodes for three-dimensional imaging," Lincoln Lab. J. 13, 335-350 (2002).

2. N. Gisin, G. G. Ribordy, W. Tittel, and H. Zbinden, "Quantum cryptography" Rev. Mod. Phys. 74, 145-195 (2002).

3. K. A. McIntosh, J. P. Donnelly, D. C. Oakley, A. Napoleone, S. D. Calawa, L. J. Mahoney, K. M. Molvar, E. K. Duerr, S. H. Groves, and D. C. Shaver, "InGaAsP/InP avalanche photodiodes for photon counting at 1.06 Mm," Appl. Phys. Lett. 81, 2505-2507 (2002).

4. B. S. Robinson, D. O. Caplan, M. L. Stevens, R. J. Barron, E. A Dauler, S. A. Hamilton, K. A. McIntosh, J. P. Donnelly, E. K. Duerr, and S. Verghese, "High-sensitivity photon-counting communications using Geiger-mode avalanche photodiodes," in 18th Annual Meeting of the IEEE Lasers and Electro-optics Soc., pp. 559-560 (2005).

5. S. Cova, M. Ghioni, A. Lacaita, C. Samori, and F. Zappa, "Avalanche photodiodes and quenching circuits for single-photon detection," Appl. Opt. 35, 1956-1976 (1996).

6. K. E. Jensen, P. I. Hopman, E. K. Duerr, E. A. Dauler, J. P. Donnelly, S. H. Groves, L. J. Mahoney, K. A. McIntosh, K. M. Molvar, A. Napoleone, D. C. Oakley, S. Verghese, C. J. Vineis, and R. D. Younger, "Afterpulsing in Geiger-mode avalanche photodiodes for 1.06 um wavelength," Appl. Phys. Lett. 88, 133503 (2006).

7. B. Moison, M. Srinivasan, and J. Hamkins, "Output Statistics of Geiger Mode APD Array, "JPL Inter-office Memo 331, Jet Propulsion Lab. (2004).

8. P. I Hopman, P. W. Boettcher, L. M. Candell, J. B. Glettler, R. Shoup, and G. Zogbi, "An end-to-end demonstration of a receiver array based free-space photon counting communications link," Proc. SPIE 6304, 63040H (2006).

9. D. M. Boroson, A. Biswas, and B. L. Edwards, "MLCD: overview of NASA's mars laser communication option," Proc. SPIE 5338, 16-28 (2004).

10. E. A. Dauler, P. I. Hopman, K. A. McIntosh, J. P. Donnelly, E. K. Duerr, R. J. Magliocco, L. J. Mahoney, K. M. Molvar, A. Napoleone, D. C. Oakley, and F. J. O'Donnell, "Scaling of dark count rate with active area in $1.06 \mu \mathrm{m}$ photon-counting in InGaAsP/InP avalanche photodiodes," Appl. Phys. Lett. 89, 111102 (2006).

11. J. P. Donnelly, E. K. Duerr, K. A. McIntosh, E. A. Dauler, D. C. Oakley, S. H. Groves, C. J. Vineis, L. J. Mahoney, K. M. Molvar, and P. I. Hopman, "Design considerations for 1.06 um InGaAsP-InP Geiger-mode avalanche photodiodes," IEEE J. Quantum Electron. 42, 797-809 (2006). 